\title{
Isothermal Desorption Hysteretic Model for Deep Coalbed Methane Development
}

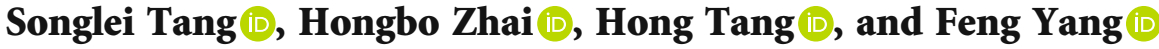 \\ Xi'an Modern Chemistry Research Institute, 710065, Xi'an, China \\ Correspondence should be addressed to Hongbo Zhai; zhaihongbo@qq.com
}

Received 10 September 2021; Revised 25 December 2021; Accepted 29 December 2021; Published 25 January 2022

Academic Editor: Kouqi Liu

Copyright (C) 2022 Songlei Tang et al. This is an open access article distributed under the Creative Commons Attribution License, which permits unrestricted use, distribution, and reproduction in any medium, provided the original work is properly cited.

\begin{abstract}
The adsorption/desorption mechanism of coalbed methane is significant for gas control and coalbed methane exploitation; scholars have done a lot of research on it and generally have confidence in that temperature, pressure, and moisture are central factors affecting the adsorption of coalbed methane. Considering the reduction of recovery efficiency caused by desorption hysteresis in deep coalbed methane drainage, the effects of high reservoir pressure, high gas content, and low permeability on the hysteresis index were analyzed. A desorption hysteresis model based on the combination of dual-porosity media and traditional Langmuir adsorption theory was proposed. By comparing with the four experimental data of Ma et al., the advantages of the new model in fitting desorption data were investigated. Based on the new desorption hysteresis model, the hysteresis index was calculated from the adsorption capacity and desorption capacity under the abandonment pressure. The hysteresis index under different coal sizes and adsorption pressure were calculated, and a good linear relationship was found between the adsorption pressure and the hysteresis index. Through a large number of field production data analysis, the following conclusions are drawn: as the adsorption pressure increases, the hysteresis index enhances; when the coal sample size increases, the hysteresis index also increases. Finally, by comparing experimental data from deep and shallow coal samples, the influence of desorption hysteresis on deep coalbed methane mining was explored. This paper draws the conclusion that although the gas content in deep coalbed methane is considerable, its hysteresis index is also enhanced, which makes coalbed methane development more difficult. The findings of this study can provide theoretical support for coal bed gas control and coal bed methane heat injection mining.
\end{abstract}

\section{Introduction}

Deep coalbed methane (CBM) resources have huge potential and are a new field for unconventional natural gas exploration and development. With the decline of shallow coalbed methane and coal resources, increasing demand for energy and intensity of mining, deep coalbed methane resources with depth of $1500 \sim 3000 \mathrm{~m}$ have gradually received attention. The development of coalbed methane has also entered a state of deep resources [1]. The increase in gas emissions from deep coal reservoirs has led to increasingly serious gas disasters, and deep coal rocks are susceptible to high geostress and pore pressure environments, and coal seam permeability is relatively low. The production of coalbed methane causes changes in pore pressure, which results in changes in coal seam skeleton stress and significant pore deformation. Therefore, the deformation of the pore space changes the movement resistance of the fluid through the pore channel, which leads to considerable change in permeability [2].

The development of coalbed methane is a dynamic process. After the confined water in the fractures is discharged, the adsorbed gas in the coal matrix desorbs flows to the pore fractures, while the free gas in the pore fractures flows to the borehole, as shown in Figure 1.

The discharge and extraction of coalbed methane lead to the reduction of gas pressure and gas concentration in the fractures. Under the effect of concentration gradient, the free gas in the matrix pores diffuses into the fractures, which promotes desorption of the gas adsorbed in the pores. In the early recognition, the process of gas adsorption and desorption in coal is generally considered to be completely reversible [3]. The 


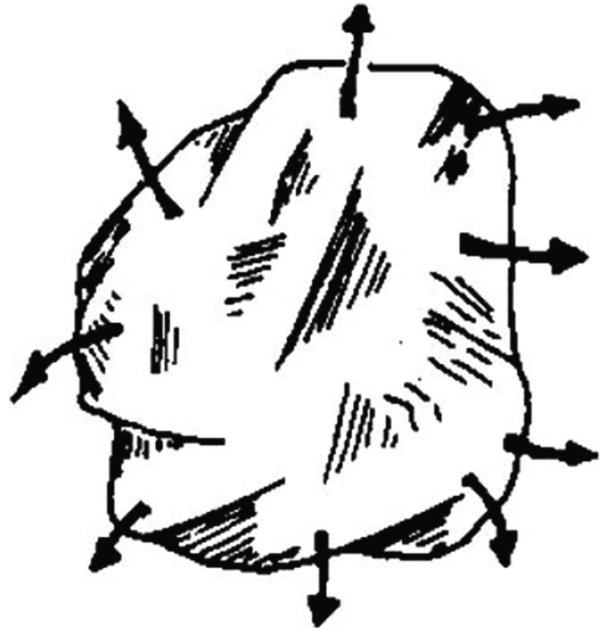

(a)

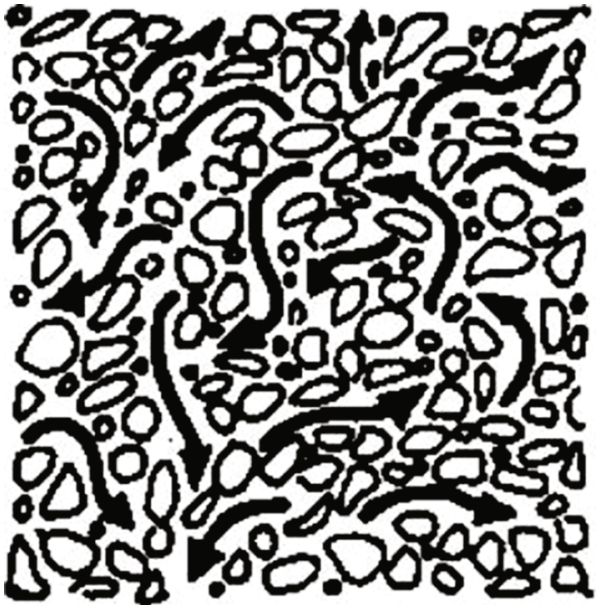

(b)

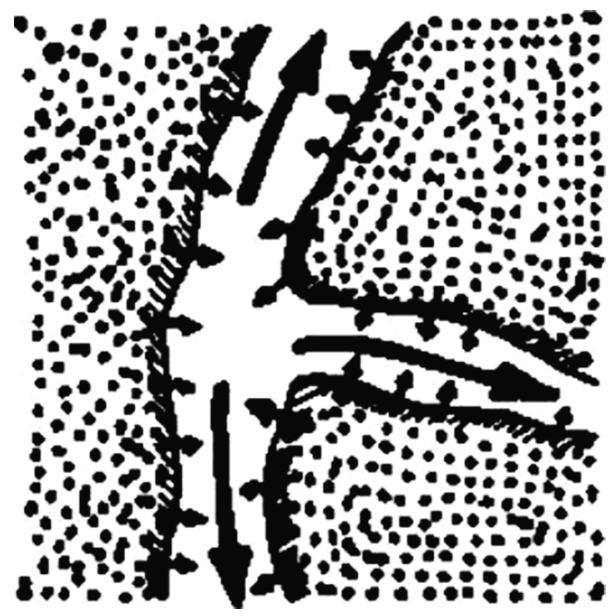

(c)

Figure 1: Schematic diagram of desorption-diffusion flow of coalbed methane [3]. (a) Desorbs from the inner surface of coal, (b) diffuses through matrix and micropores, and (c) flows in natural fracture networks.

Langmuir volume, Langmuir pressure, and recoverable reserves of coalbed methane are usually obtained by measuring the isotherm adsorption line. However, in recent years, many scholars have found that the adsorption and desorption of coalbed methane in coal are not completely reversible in most cases, and there is a hysteresis phenomenon in adsorption and desorption. As shown in Figure 2, during the adsorption and desorption cycle, the desorption gas content is always greater than the adsorption capacity under the same pressure. For deep coalbed methane, as the burial depth increases, the volatile content of coal decreases, the degree of metamorphism increases, and the gas content and gas pressure increase. As the gas pressure increases, the desorption hysteresis degree increases. In the process of deep-seated coalbed methane development, the desorption hysteresis will be more serious, which will produce larger deviations compared with the traditional reversible desorption model.

Changes in the state of the deep stress field will inevitably lead to the formation of deep coalbed methane reservoirs that vary from those of shallow formations. The gas content, gas adsorption characteristics, stress, and permeability of

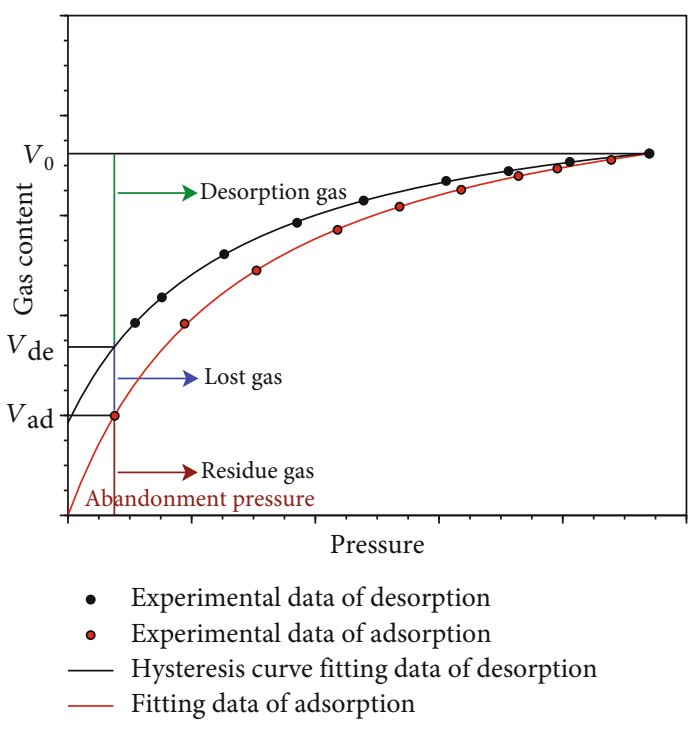

FIGURE 2: Illustration of adsorption/desorption hysteresis evaluation model. 
deep coal seams are largely different from shallow coal reservoirs: (1) coal seam gas content generally rises first and then decreases as burial depth increases; (2) logarithm of permeability has a negative linear correlation with the burial depth, that is, the burial depth of the coal seam increases, and the permeability decreases exponentially; (3) although the geostress field varies from region to region as the tectonic geological environment changes, the ratio of average horizontal stress to vertical stress decreases with increasing burial depth; (4) the total porosity of deep coal seam change is not significant compared to shallow coal seam, but the distribution of pores in the shallow is more dispersed, while the deep is more concentrated, mostly microporous.

Jessen et al. [4] found that different desorption hysteresis exists for the same coal rock sample at different maximum adsorption pressures, but the degree of hysteresis is not the same. Harpalani et al. [5] indicated that lignite in the San Juan Basin and the Illinois Basin both have coal seam gas hysteresis, but the hysteresis index is less than that of carbon dioxide. Pan et al. [6] studied coal samples in Sydney Basin with different water contents, and there was hysteresis in all the experimental groups. Zhang et al. [7] carried out isothermal desorption experiments of component gases and multicomponent gases with different ratios and proposed the relative reversibility and desorption hysteresis characteristics of the coalbed methane adsorption and desorption process. Liu et al. [8] studied the pore distribution of coal and its adsorption-desorption characteristics, and the research showed that the difference in the $\mathrm{CO}_{2} / \mathrm{CH}_{4}$ adsorption capacity is the primary cause of the phenomenon of desorption hysteresis. Song et al. [9] studied different types of deformed coal in Pingdingshan, and the experimental results showed that the adsorption and desorption of deformed coal are irreversible, and the degree of irreversible increases with the increase of coal destruction. Jian et al. [10] also found that the hysteresis of Pingdingshan pulverized coal is more obvious than that of primary structure coal. Ma et al. [11-13] studied the hysteresis phenomenon in various aspects from the perspective of CBM development, examined the effects of temperature, moisture, and other factors, and analyzed that the phenomenon does exist. It can be verified from CBM well test and drainage data that the main reason is that the micropores and pores have a strong binding capacity to gas molecules. Su et al. $[14,15]$ found that ${ }^{13} \mathrm{CH}_{4}$ has the characteristics of preferential adsorption and delayed desorption compared with ${ }^{12} \mathrm{CH}_{4}$ and explained it with the adsorption potential theory. Qiu et al. [16] found that the total heat of capillary condensation is identical to that of capillary evaporation for all systems examined, regardless of the presence of hysteresis. He et al. [17] found that original gas of three wells in the Fuling Shale in China calculated by the conventional method was lower than that calculated by the refined method, which explained the desorption hysteresis.

The above studies are only corresponding improvements based on the Langmuir model, mainly in terms of temperature, but rarely modify the adsorption analytical model in dual porous media. On this basis, four sets of coal samples from different regions are taken as the research objects. On this basis, four sets of coal samples from different regions are taken as the research objects, and the coalbed methane adsorption model under the combined action of pressure and temperature is established and revised on the highpressure coal rock matrix. However, the influence of water content on adsorptive and desorptive gas quantity is neglected in this paper, and the influence of water content needs further study.

On the basis of previous work, a quantitative evaluation index was proposed to understand the degree of hysteresis, and this index was used to evaluate the difference in the degree of adsorption and desorption hysteresis between shallow coal seams and deep coal seams. Then, on the basis of Ma's hysteresis model, a new adsorption and desorption hysteresis model was established, and the constant correction term was modified into a linear correction term to better understand the laws of adsorption and hysteresis. Finally, we attempt to explicate the mechanism of desorption hysteresis and discuss the negative effects of desorption hysteresis in deep coalbed methane mining, so as to provide guidance for engineering practice.

\section{Modified Desorption Hysteresis Model for Dual Porous Media}

Porous media with fractures and pores are called dualporous media. Fractures are mainly used as fluid flow channels, while pores are mainly used as gas storage spaces. The permeability of fractures and matrixes varies widely. According to the concept of dual-porosity medium, coal seams have a large number of micropores and fractures connecting micropores, and the permeability of pores is generally much smaller than the permeability of fractures. Therefore, most coal seams can be regarded as dual-porous media.

For the desorption hysteresis in the dual-porosity medium model, Ma et al. [13] conducted an isothermal adsorption experiment. The experimental results show that the isothermal adsorption curve conforms to the Langmuir equation, with a high degree of data fitting and small errors. The Langmuir equation of the isothermal desorption curve has a low degree of fit, indicating that the coal seam gas desorption process does not obey the Langmuir equation. Then, they proposed a modified model that included residual adsorption capacity under waste pressure. Wang et al. [18] improved the dual isothermal desorption curve of the pore model and found that it is more suitable to describe the isothermal desorption hysteresis curve. Based on Wang's model and Ma's improved model, we propose a new isothermal desorption curve. The new model assumes that the coal seam adsorbed gas volume is

$$
V=k P+C_{r}+V_{0}^{d} \frac{P}{P_{L}^{d}+P}
$$

where $k$ is Henry gas dissolution constant, $V_{0}^{d}$ and $P_{L}^{d}$ are Langmuir constants in the dual-porosity model, and $C_{r}$ is residual adsorption amounts. 
TABLE 1: Comparison table of four groups of experimental data fitting parameters.

\begin{tabular}{|c|c|c|c|c|c|c|c|c|c|c|c|}
\hline \multirow[t]{2}{*}{ Sample } & \multicolumn{2}{|c|}{$\begin{array}{l}\text { Adsorption } \\
\text { fitting } \\
\text { parameters }\end{array}$} & \multicolumn{4}{|c|}{ Desorption fitting parameters } & \multicolumn{5}{|c|}{ Improve model desorption fitting parameters } \\
\hline & $a$ & $b$ & $a$ & $b$ & $c$ & $R^{2}$ & $C_{r}$ & $k$ & $V_{0}^{d}$ & $P_{L}^{d}$ & $R^{2}$ \\
\hline Huangling $2^{\#} \mathrm{RN}$ & 18.132 & 0.233 & 17.153 & 0.215 & 1.301 & 0.999 & 1.525 & -0.289 & 23.621 & 6.615 & 0.9996 \\
\hline Zhaiyadi $9^{\#} \mathrm{JM}$ & 19.252 & 0.643 & 16.561 & 0.824 & 2.154 & 0.999 & 2.389 & -0.034 & 16.928 & 1.356 & 0.9994 \\
\hline Xiangshan $3^{\#} \mathrm{SM}$ & 20.837 & 0.297 & 16.030 & 0.322 & 3.111 & 0.998 & 0.773 & 0.402 & 12.319 & 1.116 & 0.9996 \\
\hline Sihe $3^{\#} \mathrm{WY}$ & 34.031 & 0.253 & 28.642 & 0.212 & 4.654 & 0.999 & 4.809 & -0.608 & 42.363 & 7.040 & 0.9992 \\
\hline
\end{tabular}

According to the pressure and temperature data of adsorption experiment collected by $\mathrm{Ma}$, combined with the above-mentioned improved double-pore model, the fitting degree $R^{2}$ is obtained, as shown in Table 1 .

By comparing the fitting degree $R^{2}$, the improved model has a higher fitting degree of and smaller error, which verifies the accuracy of the modified model. Since the dual porous media model is introduced into the model for understanding hysteresis, the modified model fits better. The fractures of the coal matrix satisfy the Darcy flow equation, and the micropores and nanopores satisfy the Langmuir adsorption equation.

The fitting and comparison curves of the adsorption and desorption experiment results are shown in Figure 3. When analyzing the experimental data of four different coal samples obtained by Ma et al., it was found that there were abandonment pressure points during the desorption process. The abandonment pressure point is the minimum pressure point that can be reached during the decompression and desorption process of drainage. It is impossible for the drainage decompression process to reach a state of zero pressure during the production process. Therefore, the gas content at the abandonment pressure point is an important physical quantity with a reference value.

By fitting the experimental data, the desorption effect lags behind the adsorption effect in several groups of experimental coal samples. Based on the phenomenon of adsorption hysteresis, Liu and Kang [19] defined the coalbed gas adsorption hysteresis effect as under certain conditions, the gas adsorption capacity of coal is not a single value function of matrix potential; it depends on the historical process of coalbed methane adsorption or desorption. In the practice of CBM development, affected by the hysteresis effect of coalbed methane desorption, it is necessary to use desorption isotherms instead of adsorption isotherms to evaluate gas production, gas recovery rate, recoverable reserves, and recovery efficiency.

\section{Quantitative Evaluation Index of Desorption Hysteresis}

Many scholars have proposed several evaluation indexes for different desorption hysteresis. The following sections briefly introduce several evaluation indicators.

3.1. Freundlich Index Method. The Freundlich index method decomposes the adsorption and desorption into two curves.
The adsorption process was fitted with the function $S_{\mathrm{ad}}=$ $\mathrm{K}_{\mathrm{ad}} \mathrm{Ce}_{\mathrm{ad}}^{n}$, and the desorption process with $S_{\mathrm{de}}=K_{\mathrm{de}} \mathrm{Ce}_{\mathrm{de}}^{n}$, where $S$ is the concentration of the adsorbent; Ce is the equilibrium concentration of the adsorbent; $K$ is the Freundlich adsorption parameters; $n$ is the Freundlich index; the subscripts ad and de denote the adsorption and desorption processes, respectively.

The expression of the degree of hysteresis $\mathrm{HI}$ in this irreversible segmentation process is

$$
\mathrm{HI}=\left(\frac{n_{\mathrm{ad}}}{n_{\mathrm{de}}}-1\right) \times 100 \% .
$$

The evaluation index based on the Freundlich formula was first used to indicate the concentration of adsorbed gas in the soil. It does not rely on Langmuir equations but on mathematical models in exponential form. However, the CBM content in the coal is more in line with the Langmuir curve.

3.2. Solid Phase Equilibrium Concentration Method. The solid phase equilibrium concentration method is an improvement of the Freundlich index method. The adsorption and desorption processes are still fitted with two-stage functions $S_{\mathrm{ad}}=K_{\mathrm{ad}} \mathrm{Ce}_{\mathrm{ad}}^{n}$ and $S_{\mathrm{de}}=K_{\mathrm{de}} \mathrm{Ce}_{\mathrm{de}}^{n}$, and the final evaluation index of the hysteresis becomes

$$
\mathrm{HI}=\frac{\max \left\{S_{\mathrm{ad}}-S_{\mathrm{de}}\right\}}{S_{\mathrm{ad}}} \times 100 \% .
$$

The evaluation model based on the slope and the adsorption equilibrium concentration of the solid phase describes the relative relationship between the saturated adsorption amount and the maximum desorption amount at the same temperature and pressure, but the error at this measurement point is large, and it has a great impact on the final evaluation result.

3.3. Langmuir Adsorption and Desorption Curve Area Method. The adsorption process is fitted with a Langmuir adsorption curve $V=V_{\text {ad }} P /\left(P+P_{L}\right)$, and the desorption process is fitted with a Langmuir desorption curve $V=V_{\text {de }}$ $P /\left(P+P_{L}\right)$, where $V$ represents the volume of the adsorbed gas; $P$ represents the gas pressure; $P_{L}$ represents the Langmuir pressure, which is the amount of adsorbed gas pressure at $0.5 V_{\text {ad }}$ or $0.5 V_{\mathrm{de}}$; and $V_{\text {ad }}$ and $V_{\text {de }}$ represent maximum 


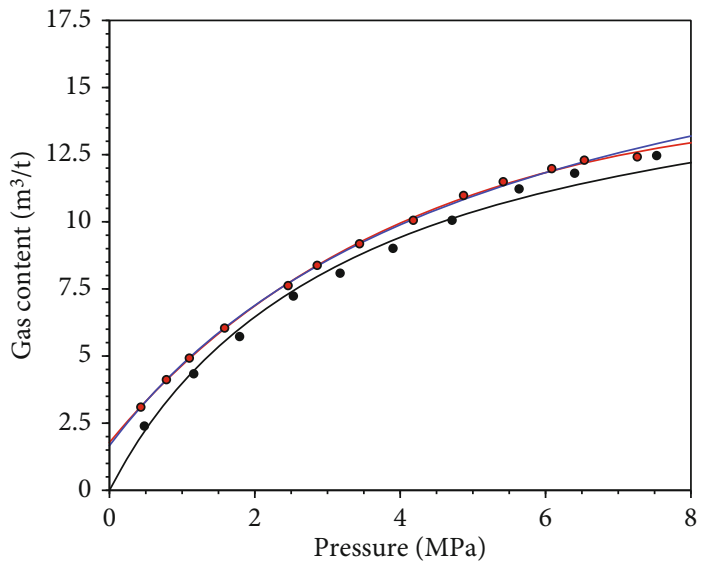

- Experimental data of adsorption

- Experimental data of desorption

- Fitting data of adsorption

- Hysteresis desorption model by Ma et al.

- Improved hysteresis desorption model

(a) Huangling $2^{\#} \mathrm{RN}$

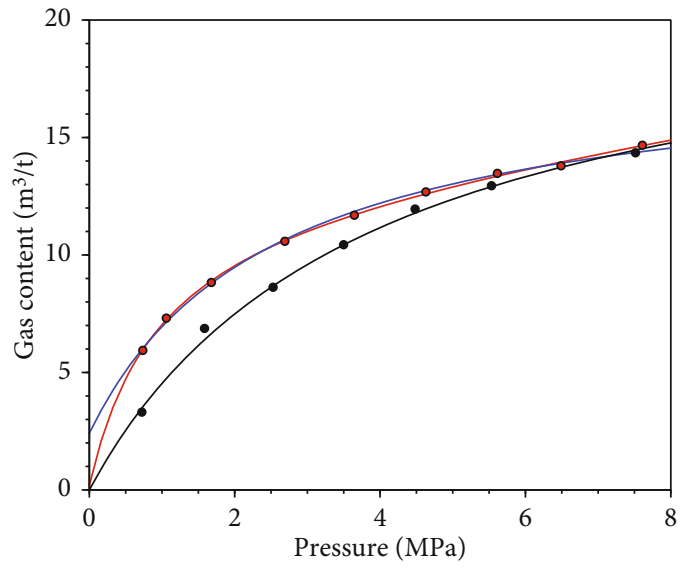

- Experimental data of adsorption

- Experimental data of desorption

- Fitting data of adsorption

- Hysteresis desorption model by Ma et al.

— Improved hysteresis desorption model

(c) Xiangshan $3^{*} \mathrm{SM}$

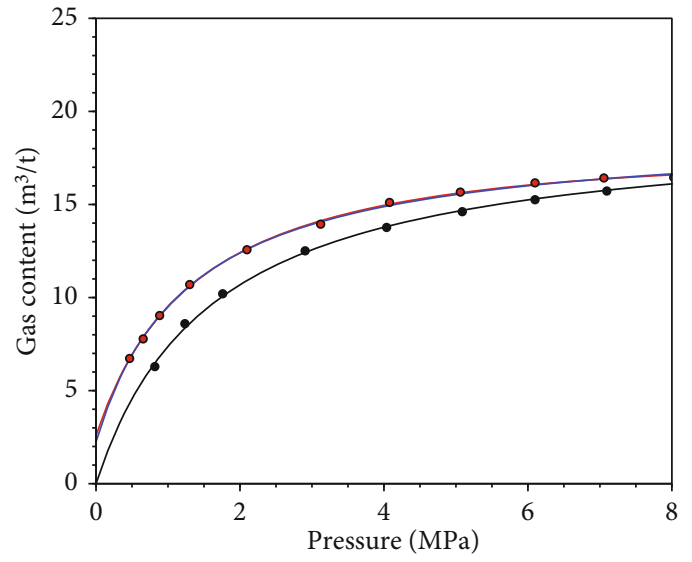

- Experimental data of adsorption

- Experimental data of desorption

- Fitting data of adsorption

- Hysteresis desorption model by Ma et al.

— Improved hysteresis desorption model

(b) Zhaiyadi $9^{\#} \mathrm{JM}$

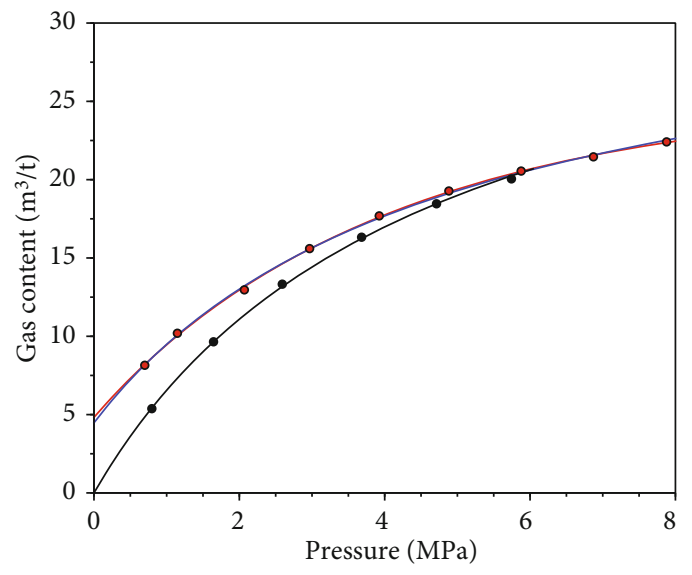

- Experimental data of adsorption

- Experimental data of desorption

- Fitting data of adsorption

- Hysteresis desorption model by Ma et al.

— Improved hysteresis desorption model

(d) Sihe $3^{\#} \mathrm{WY}$

Figure 3: Experimental data and improved fitting curves of four different coal samples.

gas adsorption and maximum desorption, respectively. The evaluation indicators of the degree of hysteresis are

$$
\mathrm{HI}=\frac{A_{\mathrm{de}}-A_{\mathrm{ad}}}{A_{\mathrm{ad}}} \times 100 \%,
$$

where $A_{\mathrm{ad}}$ and $A_{\mathrm{de}}$ are the areas under the adsorption and desorption curves. In this method, the area of the hysteresis area generated by the adsorption and desorption processes is used as the index of the degree of hysteresis.

Wang et al. [18] developed a new hysteresis evaluation model based on the area integral method. The porefractured dual-medium model Langmuir-like adsorptiondesorption curve was used to fit the isothermal adsorption test data, and then, the area of the hysteresis was used to calculate the degree of hysteresis. The new evaluation index of hysteresis is

$$
\mathrm{IHI}=\frac{A_{\mathrm{hy}}}{A_{\mathrm{hf}}}=\frac{A_{\mathrm{de}}-A_{\mathrm{ad}}}{A_{\mathrm{sf}}-A_{\mathrm{ad}}} \times 100 \%,
$$

where $A_{\text {hy }}$ is the actual measured hysteresis area; $A_{\mathrm{hf}}$ is the area of ideal nonreversible hysteresis; $A_{\mathrm{sf}}$ is the area of ideal nonreversible adsorption area.

Based on the adsorption and desorption hysteresis evaluation model by Wang et al. [18], we have made further improvements. First, use an isothermal hysteresis curve as shown in Equation (1) to fit the desorption curve. As shown 
TABLE 2: Industrial and petrographic analysis of coal sample in Sydney Basin (\%).

\begin{tabular}{|c|c|c|c|c|c|c|}
\hline \multicolumn{3}{|c|}{ Industrial analysis } & \multirow{2}{*}{ Vitrinite reflectance } & \multicolumn{3}{|c|}{ Lithofacies analysis } \\
\hline Moisture & Volatile matter & Fixed carbon & & Vitrinite group & Chitin group & Inertia group \\
\hline 1.3 & 21.7 & 71.4 & 1.28 & 41.6 & 0.1 & 55.3 \\
\hline
\end{tabular}

in Figure 2, according to the abandonment pressure point determined in the experimental data, the corresponding adsorption and desorption amount at the abandonment pressure can be calculated. The difference between the adsorption amount and the analytical amount is divided by the saturated adsorption amount to determine the coefficient of hysteresis,

$$
\mathrm{HI}=\frac{V_{\mathrm{de}}-V_{\mathrm{ad}}}{V_{0}} \times 100 \%
$$

The above-mentioned hysteresis degree coefficient reflects the desorption hysteresis coefficient under abandonment pressure. This hysteresis degree coefficient reflects the percentage of gas lost in the coal seam due to the desorption hysteresis when the downhole flow pressure reaches the abandonment pressure during the CBM drainage. The degree of hysteresis has guiding significance for the drainage and production of coalbed methane, because gas-bearing formations have a pressure limit for drainage and production, that is, the abandonment pressure. Under abandonment pressure, the degree of hysteresis directly affects the calculation of recovery factor. The only difficulty of this model is how to determine the abandonment pressure of the mine. The current mainstream abandonment pressure is $0.4 \sim 1.38 \mathrm{MPa}$, and the general compromise in the calculation process is $0.6 \mathrm{MPa}$.

Considering that the coal seam reservoir pressure at different depths is different, with the increase of the depth, the pressure of the coal seam gas reservoir will increase. Wang et al. [18] studied the methane adsorption and desorption hysteresis of coal samples in the Sydney Basin, and the industry and petrographic analysis results of coal samples in Sydney Basin are shown in Table 2. We used a modified dual-porosity medium adsorption-desorption hysteresis model to fit the data.

The experiment determined the adsorption and desorption laws of methane at three different maximum adsorption pressures $(10,20$, and $30 \mathrm{MPa}$ ) and performed filtration experiments on three groups of coal samples with different particle sizes, and the results are shown in Figure 4 . The experimental data is fitted according to Equation (1). The fitting results show the understanding of the absorption hysteresis law. It can be seen from Figure 3 that the lower the adsorption pressure, the closer the desorption curve is to the adsorption fitting curve, indicating that during the development of deep coalbed methane, the degree of coalbed methane desorption hysteresis rises. It is more difficult to reduce the pressure of drainage, and the efficiency is lower. The desorption hysteresis effect should be taken into account when predicting the recovery factor. In order to facilitate the analysis of the desorption hysteresis from the perspective of particle size, the desorption hysteresis degree $\mathrm{HI}$ of coal samples with different particle sizes was calculated separately. In order to visualize the trend, a linear fit was made to the calculation results, as shown in Figure 5.

It can be clearly seen that as the particle size of coal samples increases, the degree of desorption hysteresis of the corresponding coal samples also increases. The reason is that the hysteresis of adsorption and desorption is caused by gas molecules being embedded in micropores with poor connectivity under high pressure and unable to be desorbed from the pores. Large-particle coal samples have good integrity and contain a large number of micropores and nanopores, and there are many gas molecules that cannot be desorbed, so the relative hysteresis is relatively large.

\section{Desorption Hysteresis in Deep Coalbed Methane Mining}

In this paper, the experimental results of several groups of coal samples are fitted, and it can be found that each group of coal samples has a high desorption hysteresis in the deep environment, even up to $10 \%$. The adsorption and desorption processes of deep coal samples are two completely different processes. In the process of coal diagenesis for millions of years, coalbed methane has continuously accumulated and adsorbed in the coal matrix. Therefore, the coalbed methane mining process is a very fast process; the desorption process can be completed in only about ten years, so the disturbance of the coal matrix during the desorption process is more severe. The coalbed methane adsorption takes place in a long process of coalification, which is a very complicated physicochemical process. In contrast, there is an essential difference between the passive desorption of coalbed methane and the adsorption process in the drainage and depressurization process. During the passive desorption process, the gas adsorbed in the pores quickly desorbs and escapes, causing irreversible damage to the coal sample. In the process of coalbed methane exploitation, greater irreversible damage will be caused due to greater formation pressure. Gas molecules are embedded in micropores with poor connectivity under high pressure and cause pore deformation. The adsorbed gas molecules are restricted by narrow pore channels and cannot be desorbed from the pores, resulting in desorption hysteresis.

The difference in porosity of deep and shallow reservoir coal seams is also an important reason for the hysteresis phenomenon of adsorption and desorption. As mentioned earlier, there is no significant difference in the total porosity of deep coal rocks and shallow coal rocks, but the distribution of pores in the shallow parts is more dispersed, while the deep parts are more concentrated, mostly micropores 


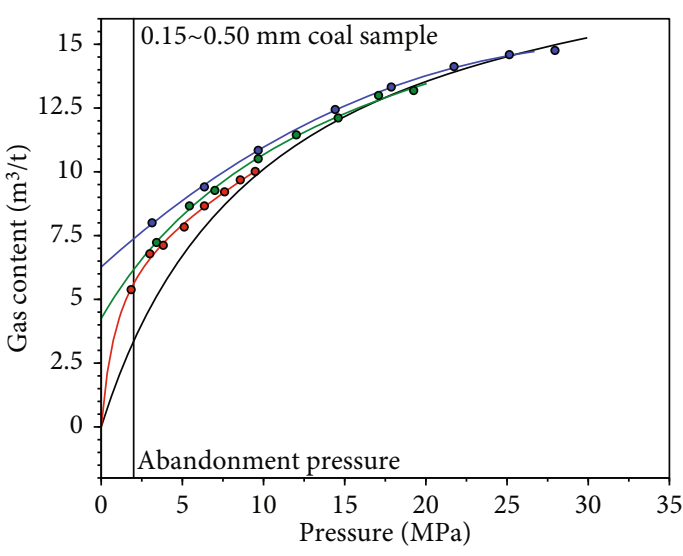

- Maximum adsorption pressure $10 \mathrm{MPa}$

- Maximum adsorption pressure $20 \mathrm{MPa}$

- Maximum adsorption pressure $30 \mathrm{MPa}$

$10 \mathrm{MPa}$ fitted curve

- $20 \mathrm{MPa}$ fitted curve

$30 \mathrm{MPa}$ fitted curve

- Langmuir adsorption curve

(a) Experimental data of $0.15 \sim 0.50 \mathrm{~mm}$ coal sample

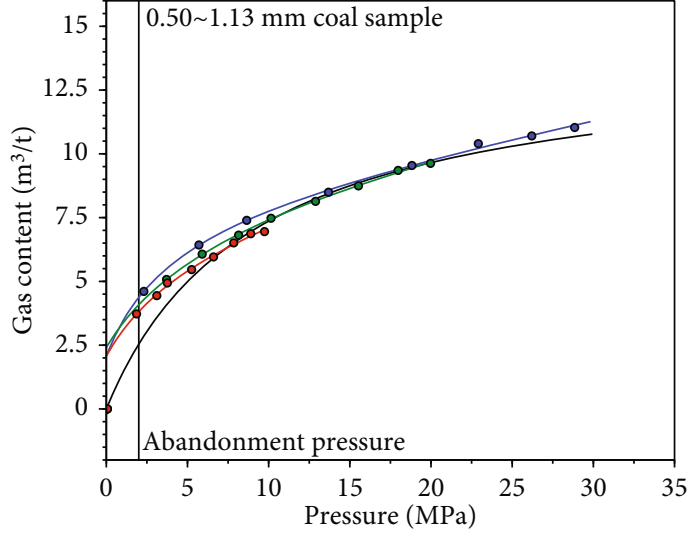

- Maximum adsorption pressure $10 \mathrm{MPa}$

- Maximum adsorption pressure $20 \mathrm{MPa}$

- Maximum adsorption pressure $30 \mathrm{MPa}$

$10 \mathrm{MPa}$ fitted curve

$20 \mathrm{MPa}$ fitted curve

$30 \mathrm{MPa}$ fitted curve

- Langmuir adsorption curve

(b) Experimental data of $0.50 \sim 1.13 \mathrm{~mm}$ coal sample

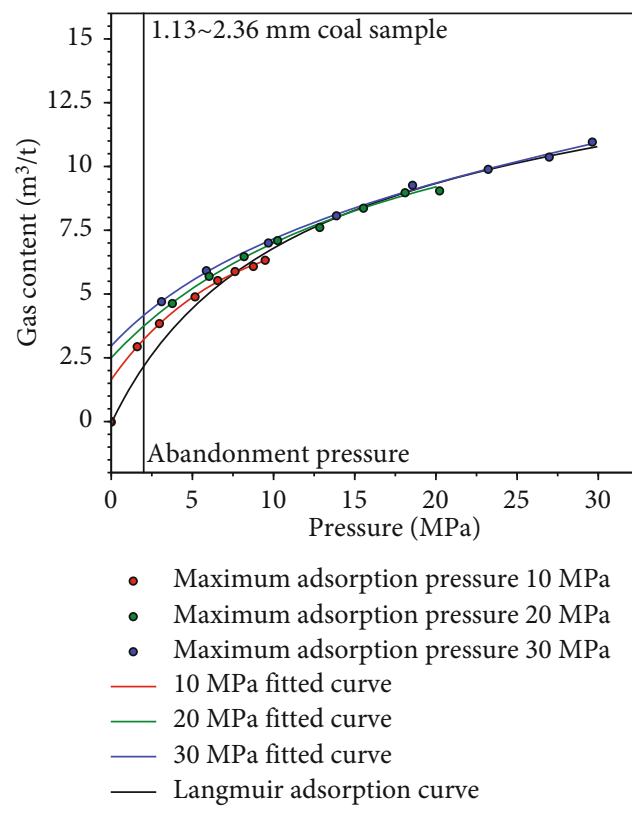

(c) Experimental data of $1.13 \sim 2.26 \mathrm{~mm}$ coal sample

FIgURE 4: Comparison of isothermal desorption lines of methane at different highest adsorption pressures.

and nanopores. According to the definition of the International Association of Pure and Applied Chemistry (IUPAC), the diameter of a micropore is a pore structure smaller than $50 \mathrm{~nm}$. Although the molecular diameter of methane gas molecules is smaller than the diameter of the micropores, the interaction between molecules makes it extremely tough to enter the micropores. When the storage pressure is relatively low, there are a small amount of methane molecules in the micropores, but as the storage pressure increases, the amount of methane gas stored in the micropores increases. Because the permeability of the pores is deteriorated during the adsorption process, the gas adsorbed during the desorption process cannot escape, resulting in the hysteresis phenomenon of adsorption and desorption. Under the action of deep high pressure, the number of gas molecules that can be contained in the pores of coal rocks increases, and in addition, the gas molecules can be embedded in the ultramicropores in the coal that could not be accessed under the original low-pressure conditions, which in turn exacerbates the phenomenon of pore expansion and deformation, resulting in more pronounced adsorption hysteresis.

Compared with shallow coal seams, deeper coal seams have higher temperature, higher metamorphism, and higher 


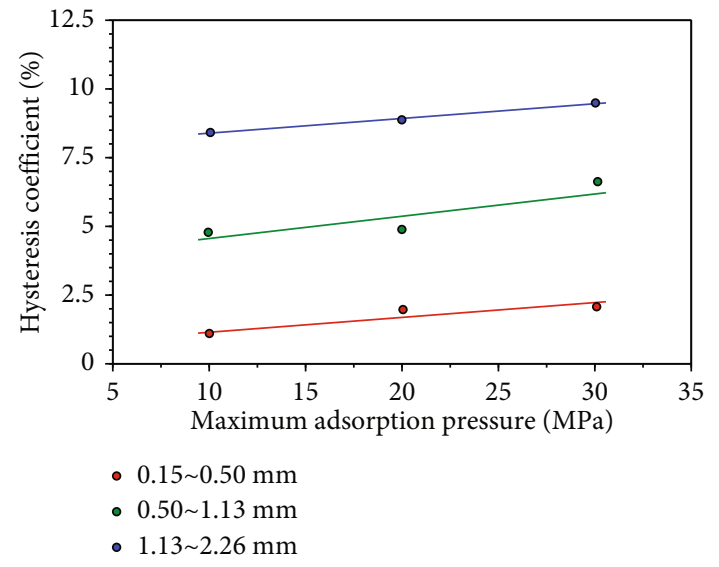

Figure 5: Degree of hysteresis and relationship between maximum adsorption pressure and coal particle size.

gas pressure, which is one of the reasons for the hysteresis of adsorption and desorption. As the gas pressure increases and the degree of coal rock metamorphism increases, the proportion of micropores in the coal body increases, the possibility of micro- and nanopore sizes further increases, and the degree of desorption hysteresis increases.

It can be inferred that the gas content of deep coalbed methane is higher than that of shallow coalbed methane, but affected by the phenomenon of desorption hysteresis, it is more difficult for the gas adsorbed in the pores to move from the pores to the fractures. The outflow rate may be a factor restricting the development of deep coalbed methane. Therefore, under the combined effect of other characteristics such as adsorption and desorption hysteresis and low permeability caused by high geostress, the difficulty of mining deep coalbed methane increases. Making the development of coalbed methane fail to reach the expected output caused huge economic losses.

\section{Summary and Conclusions}

(1) A quantitative evaluation index of the degree of desorption hysteresis based on the theory of dualporosity media was proposed in this paper, which can accurately reflect the irreversibility of desorption under abandonment pressure. The accuracy of the new model was verified by fitting and comparison with the experimental data of adsorption and desorption by $\mathrm{Ma}$ et al. The proposed improved evaluation index was used to calculate the coal rock test results in the Sydney Basin. The results show that the higher the adsorption pressure, the greater the hysteresis; the larger the coal particle size, the stronger the hysteresis

(2) In the passive desorption process, the gas adsorbed in the pores quickly desorbs and escapes, causing irreversible damage to the coal sample and causing pore deformation, which is an important factor causing the hysteresis phenomenon of desorption
(3) There is no significant difference in the total porosity of deep coal rocks and shallow coal rocks, but the proportion of micro- and nanopores in deep coal rocks is high. The existence of micro- and nanopore and methane molecular diameters in the coal body, and only high-pressure gas can enter into it, resulting in deformation of the coal body and expansion from the inside to the outside, which eventually increases the degree of desorption hysteresis

(4) With the combined effects of high geostress and low permeability, the hysteresis phenomenon of desorption is more significant, and the difficulty of mining deep coalbed methane will increase, making the development of coalbed methane less than the expected output, resulting in huge economic losses. Affected by the coal seam gas desorption hysteresis effect, the desorption isotherm is needed to evaluate the gas production capacity, gas production rate, recoverable reserves, and recovery factor in the development of coal seam gas

\section{Nomenclature}

$V: \quad$ Gas volume

$V_{\text {ad }}:$ Volume of adsorbed gas

$V_{\mathrm{de}}$ : Volume of desorbed gas

$k$ : Henry gas dissolution constant

$P: \quad$ Gas pressure

$C_{r}: \quad$ Residual adsorption amounts

$V_{0}^{d}$ : Langmuir volume constants

$P_{L}^{d}$ : $\quad$ Langmuir pressure constants

$R^{2}$ : $\quad$ Fitting degree

$S_{\text {ad }}$ : Adsorption concentration of the adsorbent

$K_{\text {ad }}$ : Freundlich adsorption parameters

$\mathrm{Ce}_{\mathrm{ad}}^{n}$ : Adsorption equilibrium concentration

$S_{\mathrm{de}}$ : Desorption concentration of the adsorbent

$K_{\mathrm{de}}:$ Freundlich desorption parameters

$\mathrm{Ce}_{\mathrm{de}}^{n}$ : Desorption equilibrium concentration

HI: Hysteresis index

$n_{\mathrm{ad}}:$ Mass of adsorbed gas

$n_{\mathrm{de}}: \quad$ Mass of desorbed gas

$P_{L}$ : Langmuir pressure

$A_{\text {ad }}$ : Areas under the adsorption curves

$A_{\text {de }}:$ Areas under the desorption curves

$A_{\text {hy }}$ : Actual measured hysteresis area

$A_{\mathrm{hf}}:$ Area of ideal nonreversible hysteresis

$A_{\mathrm{sf}}$ : Area of ideal nonreversible adsorption area.

\section{Data Availability}

The data that supports the findings of the study are available in the supplementary material of this article.

\section{Conflicts of Interest}

No potential conflict of interest was reported by the authors. 


\section{Acknowledgments}

This project is granted by the National Natural Science Foundation of China (41472130).

\section{References}

[1] S. Li, D. Tang, H. Xu, and T. Shu, "Progress in geological researches on the deep coalbed methane reservoirs," Earth Science Frontiers, vol. 23, no. 3, pp. 10-16, 2016.

[2] M. He, H. Xie, S. Peng, and Y. Jiang, "Study on rock mechanics in deep mining engineering," Chinese Journal of Rock Mechanics and Engineering, vol. 24, no. 16, 2005.

[3] W. Zhang, Y. Yan, X. Kong et al., "Structure and composition characteristics of deformed high-rank coals in the south of Qinshui basin and their influence on CBM adsorption/desorption," Journal of University of Chinese Academy of Sciences, vol. 31, no. 1, pp. 98-107, 2014.

[4] K. Jessen, G. Tang, and A. Kovscek, "Laboratory and simulation investigation of enhanced coalbed methane recovery by gas injection," Transport in Porous Media, vol. 73, no. 2, pp. 141-159, 2008.

[5] S. Harpalani, B. Prusty, and P. Dutta, "Methane/ $\mathrm{CO}_{2}$ sorption modeling for coalbed methane production and $\mathrm{CO}_{2}$ sequestration," Energy \& Fuels, vol. 20, no. 4, pp. 1591-1599, 2006.

[6] Z. Pan, L. Connell, M. Camilleri, and L. Connelly, "Effects of matrix moisture on gas diffusion and flow in coal," Fuel, vol. 89, no. 11, pp. 3207-3217, 2010.

[7] S. Zhang, J. Ye, S. Tang, D. Ma, and Y. Huo, "Theoretical analysis of coal-methane adsorption/desorption mechanism and its reversibility experimental study," Natural Gas Industry, vol. 25, no. 1, pp. 44-46, 2005.

[8] G. Liu, Z. Zhang, X. Zhang, and R. S. Lu, "Pore distribution regularity and absorption-desorption characteristics of gas coal and coking coal," Chinese Journal of Rock Mechanics and Engineering, vol. 28, no. 8, pp. 1587-1590, 2009.

[9] Z. Song, G. Liu, X. Yang, and Z. X. Zhang, "Adsorptiondesorption characteristic on deformed coal under the conditions of high temperature and pressure and equilibrium water," Journal of Mining and Safety Engineering, vol. 29, no. 4, pp. 591-595, 2012.

[10] F. Jian Huo and Z. Y. Xuehai, "Analysis on coalbed methane desorption stage and calculation on max instant desorption value of tectonic coal," Coal Science and Technology, vol. 43, no. 4, pp. 57-62, 2015.

[11] M. Dongmin, L. Laixin, L. Xiaoping et al., "Contrastive experiment of adsorption-desorption between $\mathrm{CH}_{4}$ and $\mathrm{CO}_{2}$ in coal seam 4 of Dafosi coal mine," Journal of Coal, vol. 39, no. 9, pp. 1938-1944, 2014.

[12] M. Dongmin, M. Wei, and L. Yabin, "Desorption hysteresis characteristics of CBM," Journal of China Coal Society, vol. 37, no. 11, pp. 1885-1889, 2012.

[13] M. Dongmin, Z. Suian, and L. Yabin, "Isothermal adsorption and desorption experiment of coal and experimental results accuracy fitting," Journal of China Coal Society, vol. 36, no. 3, pp. 477-480, 2011.

[14] S. Xianbo, C. Run, L. Xiaoying, and J. H. Li, "Desorption characteristic curves of carbon dioxide and methane in coal and their applica-tion," Natural Gas Industry, vol. 28, no. 7, pp. 17-19, 2008.
[15] S. Xianbo, C. Run, L. Xiaoying, and Y. Song, "Application of adsorption potential theory in the fractionation of coalbed gas during the process of adsorption/desorption," Acta Geologica Sinica, vol. 82, no. 10, pp. 1382-1389, 2008.

[16] Q. Xingdong, Y. Huan, D. Morteza, P. Tan Sugata, and A. Hertanto, "Experiments on the capillary condensation/ evaporation hysteresis of pure fluids and binary mixtures in cylindrical nanopores," The Journal of Physical Chemistry C, vol. 125, no. 10, pp. 5802-5815, 2021.

[17] L. He, H. Mei, X. Hu, M. Dejam, Z. Kou, and M. Zhang, "Advanced flowing material balance to determine original gas in place of shale gas considering adsorption hysteresis," SPE Reservoir Evaluation \& Engineering, vol. 22, no. 4, pp. 1282-1292, 2019.

[18] W. Gongda, R. Tingxiang, Q. Qingxin, K. Wang, and L. Zhang, "Mechanism of adsorption-desorption hysteresis and its influence on deep CBM recovery," Journal of China Coal Society, vol. 41, no. 1, pp. 49-56, 2016.

[19] L. Xianzhao and K. Shaozhong, "Hysteresis in process of rainfall in filtration-runoff on hill slope in loess area," Advances in Water Science, vol. 12, no. 1, pp. 56-60, 2001. 